\title{
Condicionantes ambientais na distribuição e no periodo reprodutivo do Orthopristis ruber (Cuvier) (Teleostei, Haemulidae) na Baía de Sepetiba, Rio de Janeiro, Brasil
}

\author{
André L. B. dos Santos; André L. M. Pessanha; Francisco G. Araújo \& Marcus R. da Costa
}

Laboratório de Ecologia de Peixes, Universidade Federal Rural do Rio de Janeiro. Antiga Rio São Paulo, km 47, 23851-970 Seropédica, Rio de Janeiro, Rio de Janeiro,Brasil.E-mail: gerson@ufrrj.br; albs03@ufrrj.br

\begin{abstract}
Environmental constraints on distribution and reproductive period of Orthopristis ruber (Cuvier) (Teleostei, Haemulidae) in the Sepetiba Bay, Rio de Janeiro, Brasil. Orthopristis ruber (Cuvier, 1830) is a very common species in the Brazilian coast, being found in large abundance in bays where they rank among the most numerical abundant species in bottom trawls. This work aims to assess environmental influences on spatial, temporal distribution and reproductive period of 0 . ruber in the Sepetiba Bay, a coastal semi-closed area in the Rio de Janeiro State. Monthly samplings were carried out using otter trawl from October 1998 to September 1999, in three Bay zones, defined according to the proximity of the sea (inner, middle and outer). Samples were taken as triplicate at each site as well as environmental measurements of water temperature, salinity, transparency and depth. Relative abundance was assessed by CPUE (capture per unit effort) in both number and weight. The reproductive period was determined by the gonadossomatic index. A total of 866 individuals was caught, with size ranging from 31 to $293 \mathrm{~mm}$ Total Length and sex ratio 1:1. Higher fish numbers and biomass were recorded in January/February and May, although no significant difference was found among seasons. Spatially, the highest number and biomass were recorded in the outer zone. Positive significant correlation between relative abundance and depth, salinity and transparency and negative with temperature were found. The reproductive period, indicated by the highest gonadosomatic index (IGS) occurred probably between June and September, coinciding with the lowest temperatures. This species seems to have distribution limited to outer bay zones, near to the sea influence, with the highest influxes in Summer, as a probable effect of the South Atlantic Central Waters (SACW) that compress Coastal Waters (CW) toward the bay.

KEY WORDS. Bays; coastal fishes; environmental variables; fish ecology; grunts.
\end{abstract}

RESUMO. Orthopristis ruber (Cuvier, 1830) é uma espécie muito comum na costa brasileira, sendo encontrada em baias, onde figura entre os mais abundantes peixes nos arrastos de fundo. Este trabalho tem por objetivo avaliar a influência das condicionantes ambientais na distribuição espaço-temporal e no período reprodutivo de 0 . ruber na Baía de Sepetiba, uma área costeira semi-fechada no sul do Rio de Janeiro. Foi realizado um programa de arrasto de fundo, entre outubro de 1998 e setembro de 1999, em três zonas da baía, estabelecidas de acordo com sua proximidade do mar (interna, central e externa). Amostras mensais de peixes foram tomadas em triplicatas em cada ocasião de amostragem, bem como foram medidas as variáveis ambientais de temperatura, salinidade, transparência e profundidade. Abundância relativa foi avaliada com base nas CPUEs (Captura por Unidade de Esforço) em termos de número e de biomassa, enquanto o período reprodutivo foi determinado com o índice gonadossomático. Um total de 866 indivíduos foi capturado, com o tamanho variando entre 31 e $293 \mathrm{~mm}$ de comprimento total e proporção sexual de 1:1. Um maior número de peixes foi registrado em janeiro/fevereiro e em maio, embora não tenha sido encontrada diferença na abundancia relativa entre as estações do ano. Espacialmente, os maiores números e biomassas foram registrados na zona externa. Correlação positiva significativa foi encontrada entre a abundancia relativa e a profundidade, salinidade e transparência, e negativa com a temperatura. $O$ período reprodutivo, indicado pelos maiores valores de índices gonadosomático (IGS), provavelmente ocorre de junho a setembro, coincidindo com as menores temperaturas. Esta espécie parece ter distribuição limitada às zonas mais externa da baia, próxima à influência com o mar, com maiores influxos no verão, como provável efeito da das Águas Centrais do Atlântico Sul (ACAS) que comprimem as Águas Costeiras (AC) para a baía. PALAVRAS-CHAVE. Baias; cocoroca; ecologia de peixes; peixes costeiros; variáveis ambientais. 
Os peixes conhecidos como cocorocas, pertencente à família Haemulidae, e ocorrem nos mares tropicais e subtropicais, sendo amplamente distribuídos na costa brasileira Menezes \& Figueiredo (1980). Na Baía de Sepetiba, uma área semi-fechada da costa do Estado do Rio de Janeiro, com cerca de $520 \mathrm{~km}^{2}$ de espelho d'água, a espécie Orthopristis ruber (Cuvier, 1830) é o mais abundante representante desta família e tem se destacado pôr suas elevadas contribuições nas amostras de arrasto de fundo (Araújo et al. 2002).

Alguns aspectos são de grande importância para o ciclo de vida das espécies marinhas, que utilizam zonas costeiras semifechadas visando tirar proveito dos recursos disponíveis e obter sucesso ao longo das gerações. Neste escopo, os movimentos que definem mudanças nas abundancias relativas espaçotemporal, os períodos e locais de reprodução e as áreas de alimentação são determinados ao longo da história evolutiva das espécies, por condicionantes ambientais e interações bióticas (Weinstein 1982, Wootton 1992, Helfman et al. 2000).

É de amplo conhecimento que muitas interações de fatores físicos e biológicos influenciam a ocorrência, distribuição, abundancia e diversidade de peixes estuarinos tropicais (Albaret 1999, BLABER 2000), citando-se entre os fatores mais importantes a salinidade, temperatura, turbidez, profundidade, força da corrente, oxigênio dissolvido e a flutuabilidade em diferentes escala do tempo. Deste modo, os peixes permanecem ou deslocamse para dentro ou para fora de um determinado ambiente quando tais condições lhes são favoráveis (MoyLE \& CECH 1988). Neste movimento, ditado muitas vezes pela procura de áreas de reprodução e de alimentação, são importantes o conhecimento de indicadores desta fase da vida dos peixes como o índice gonadosomático, indicador do período reprodutivo, e o fator de condição, indicação do estado de "bem-estar" do peixe.

O conhecimento do período reprodutivo é um aspecto essencial da estratégia reprodutiva das espécies, que constitui um conjunto de características que uma espécie manifesta para ter sucesso ao longo de gerações, de modo a garantir o equilíbrio da população (VAzzoler 1996). Neste aspecto, o índice gonadossomático constitui um importante parâmetro para avaliação do período reprodutivo dos peixes, já que expressa o desenvolvimento das gônadas, sendo um indicador eficiente do estado funcional dos ovários (IsAaC-NAHUM \& VAzzoler 1987, Wooton 1989, VAzZoler 1996).

O fator condição (Le CREN 1951) é um indicador quantitativo do bem estar do peixe, refletindo condições alimentares recentes, além de ser útil como indicador do acúmulo de gordura, e da massa corporal, servindo para indicar investimentos energéticos para crescimento e ou reprodução, fenômenos normalmente cíclicos nas populações de peixes.

Poucas são as informações disponíveis na literatura sobre aspectos do ciclo de vida de $O$. ruber em baias, especialmente no que se refere às influencias ambientais na abundância e no período reprodutivo. Este trabalho tem por objetivo caracterizar a distribuição espaço-temporal desta espécie e o período reprodutivo, bem como avaliar possíveis associações com as variáveis ambientais, contribuindo assim para o conhecimento deste abundante recurso na Baía de Sepetiba.

\section{MATERIAL E MÉTODOS}

\section{Área de estudo}

A Baía de Sepetiba (Fig. 1) está localizada no Sudeste do Estado do Rio de Janeiro $\left(22^{\circ} 54^{\prime}-23^{\circ} 04^{\prime}\right.$ S; $\left.43^{\circ} 34^{\prime}-44^{\circ} 10^{\prime} \mathrm{W}\right)$. Apresenta uma área de aproximadamente $520 \mathrm{~km}^{2}$, limitandose ao Norte e Leste pelo continente, ao Sul pela Restinga de Marambaia e a Oeste, pela Baía de Ilha Grande. Seu maior comprimento é de $43 \mathrm{~km}$ no sentido leste-oeste e sua maior largura é de $17 \mathrm{~km}$ no sentido norte-sul, com perímetro de aproximadamente $123 \mathrm{~km}$. A bacia hidrográfica apresenta área aproximada de $1800 \mathrm{~km}^{2}$, incluindo a Restinga de Marambaia e ilhas no interior da baia. As cotas altimétricas da bacia variam de 0 a $1800 \mathrm{~m}$, sendo o ponto culminante na Serra do Couto. Em termos geológicos-geomorfologicos, a bacia hidrográfica da baia de Sepetiba apresenta dois conjuntos fisiograficos distinto: o domínio Serrano representado por montanhas e escarpas da vertente oceânica da Serra do Mar, maciços costeiros (Pedra Branca, Mendanha e Ilha da Marambaia); e o Domino da baixada, representado por uma extensa planície flúvio-marinha, atravessada por rios que desembocam na baia de Sepetiba.

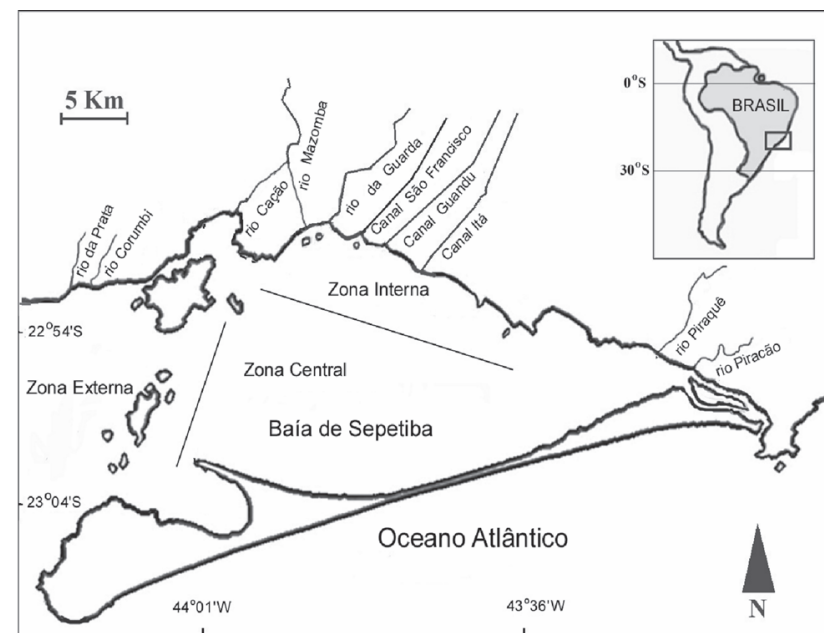

Figura 1. Área de estudo, Baía de Sepetiba', com indicação das três zonas de coletas.

\section{Métodos}

Foram analisados dados das amostragens de arrasto de fundo na Baía de Sepetiba, entre outubro de 1998 e setembro de 1999, utilizando-se um barco arrasteiro provido de redes de arrasto com portas: tralha superior $=10,5 \mathrm{~m}$; tralha inferior $=12$ $\mathrm{m}$; malha de $25 \mathrm{~mm}$ entre os nós consecutivos nas asas; $15 \mathrm{~mm}$ na região do ensacador. Foram feitas amostragens em triplicata em cada zona, (interna, central e externa) em locais escolhidos 
aleatoriamente (Fig. 1). Cada arrasto padronizado com $30 \mathrm{mi}$ nutos de duração e velocidade de dois nós, correspondendo a aproximadamente $1 \mathrm{~km}$ de arrasto em linha reta. Em cada amostragem foram retirados os parâmetros ambientais de temperatura da água, salinidade, transparência da água e profundidade. A temperatura da água de fundo foi aferida com um sensor da marca Horiba; para salinidade um salinômetro; a transparência da água foi obtida com o uso do disco de Secchi com graduação em centímetros, e para determinar a profundidade foi utilizado um profundímetro com graduação em centímetros. Os dados abióticos de pluviometria foram fornecidos pelo Departamento de Metereologia do Ministério da Agricultura, Regional do Rio de Janeiro.

Três zonas foram estabelecidas para comparações espaciais na área de estudo, visando descrever os gradientes ambientais: zona interna, com localização na região mais protegida da baía, com maior influencia de aporte fluvial; zona externa, com localização na região de comunicação direta com as águas oceânicas; e zona central, com características intermediárias e localizadas entre as duas zonas anteriores (Fig. 1).

Os peixes coletados foram fixados em formalina a 10\%, e conduzidos a laboratório, onde foram preservados em álcool 70\% após 48 horas. A identificação foi feita segundo MenEzes \& FigueIREdo (1980), com cada indivíduo tendo sido medido o comprimento total (em milímetros) e pesado com balança de precisão de 0,1 g. A distribuição e a abundância relativa da espécie foram calculadas com base nas CPUEs (indivíduos/arrasto) e (peso/arrasto), com as variações espaciais tendo sido feitas considerando as zonas de coleta, e as variações sazonais considerando os meses de amostragem agrupados por estação do ano: primavera: outubro, novembro e dezembro; verão: janeiro, fevereiro e março; outono: abril, maio e junho; e inverno: julho, agosto e setembro.

$\mathrm{O}$ fator de condição $(\mathrm{K})$ foi determinado para os sexos em separado, através da equação: $\mathrm{K}=\mathrm{P}_{\mathrm{T}} / \mathrm{C}_{\mathrm{T}}$; ; onde $\mathrm{P}_{\mathrm{T}}$ é o peso total (g), $\mathrm{C}_{\mathrm{T}}$ o comprimento total ( $\left.\mathrm{mm}\right)$, e b o coeficiente de alometria obtido da relação peso-comprimento que foi determinada por
SANTOS et al. (2004). O índice gonadossomático (IGS) foi calculado através da equação: IGS $=\left(\mathrm{Pg} / \mathrm{P}_{\mathrm{T}}\right){ }^{*} 100$, onde Pg é o peso das gônadas (g), e $P_{\mathrm{T}}$ é o peso total do indivíduo, (VAzzoler 1996).

Para detectar diferenças entre os valores médios dos dados abióticos (parâmetros ambientais) e bióticos (CPUEs, K e IGS), foi utilizada a Análise de Variância (ANOVA) ao nível de confiança de $95 \%(\mathrm{p}<0,05)$, seguida do teste a posteriori de Tukey. Os valores dos fatores abióticos, CPUEs (indivíduos e peso) sofreram prévia transformação logarítmica $\left[\log _{10}(X+1)\right]$ para atender aos requisitos de normalidade e homocedasticidade. Foi aplicado o teste do Qui-quadrado para testar a proporção sexual por classes de tamanho com os grupos de comprimento em classes de $20 \mathrm{~mm}$. O coeficiente de correlação de Spearman foi utilizado para medir a associação entre os parâmetros ambientais e as CPUEs (indivíduos e peso).

\section{Parâmetros ambientais}

\section{RESULTADOS}

Os maiores valores de precipitação ocorreram no verão, seguidos da primavera, e os menores no outono e inverno (Fig. 2). No entanto, diferenças significativas não foram detectadas pela ANOVA devido a grande variabilidade dos dados $(\mathrm{F}=0,96$, $\mathrm{p}=0,45)$

A temperatura apresentou valores médios entre $21,0^{\circ}$ e $27,5^{\circ} \mathrm{C}$. Sazonalmente os maiores valores foram registrados no verão e os menores na primavera/outono (Fig. 2, Tab. I). Espacialmente os maiores valores da temperatura da água foram registrados na zona interna (ZI) e os menores valores na zona externa (ZE) para a primavera e verão, enquanto no outono e inverno não foram observadas estas diferenças, indicadas pelas interações significativas zona versus estação do ano (Fig. 2, Tab. I).

A salinidade apresentou valores médios entre 28,5 e 34,5. Sazonalmente os maiores valores foram registrados na primavera/verão e os menores valores foram registrados no outono/ inverno (Tab. I). Espacialmente os maiores valores foram registrados na zona externa (ZE) e os menores valores na zona interna (ZI) (Fig. 2, Tab. I).

Tabela I. Valores e significância de F da Análise de Variância para as comparações dos parâmetros ambientais na Baía de Sepetiba, Rio de Janeiro, 1998/1999. Zonas da Baía: (ZI) zona interna, (ZC) zona central, (ZE) zona externa. Estações do ano: (PRI) primavera, (VER) verão, (OUT) outono, (INV) inverno.

\begin{tabular}{|c|c|c|c|c|}
\hline \multirow{2}{*}{ Parâmetros } & \multicolumn{3}{|c|}{ Fator de Variação } & \multirow{2}{*}{ Teste de Tukey } \\
\hline & Zona & Estações do Ano & Interação $1 \times 2$ & \\
\hline Pluviosidade & - & ns & - & - \\
\hline \multirow[t]{2}{*}{ Temperatura } & $4,65^{*}$ & $26,72^{\star *}$ & $5,16^{* *}$ & $\mathrm{ZI}>\mathrm{ZE}$ \\
\hline & & & & VER $>$ PRI,OUT,INV \\
\hline \multirow[t]{2}{*}{ Salinidade } & $37,77^{\star *}$ & $5,98^{* *}$ & ns & $\mathrm{ZE}>\mathrm{ZC}>\mathrm{ZI}$ \\
\hline & & & & PRI,VER > OUT,INV \\
\hline Profundidade & $248,17^{* *}$ & $\mathrm{~ns}$ & $\mathrm{~ns}$ & $Z E>Z C>Z I$ \\
\hline Transparência & $80,15^{\star *}$ & $\mathrm{~ns}$ & ns & $Z E>Z C>Z I$ \\
\hline
\end{tabular}

* Significativo ao nível de $95 \%$ de probabilidade $(p<0,05)$; ** significativo ao nível de $99 \%$ de probabilidade $(p<0,01)$.

Revista Brasileira de Zoologia 24 (4): 1017-1024, dezembro 2007 

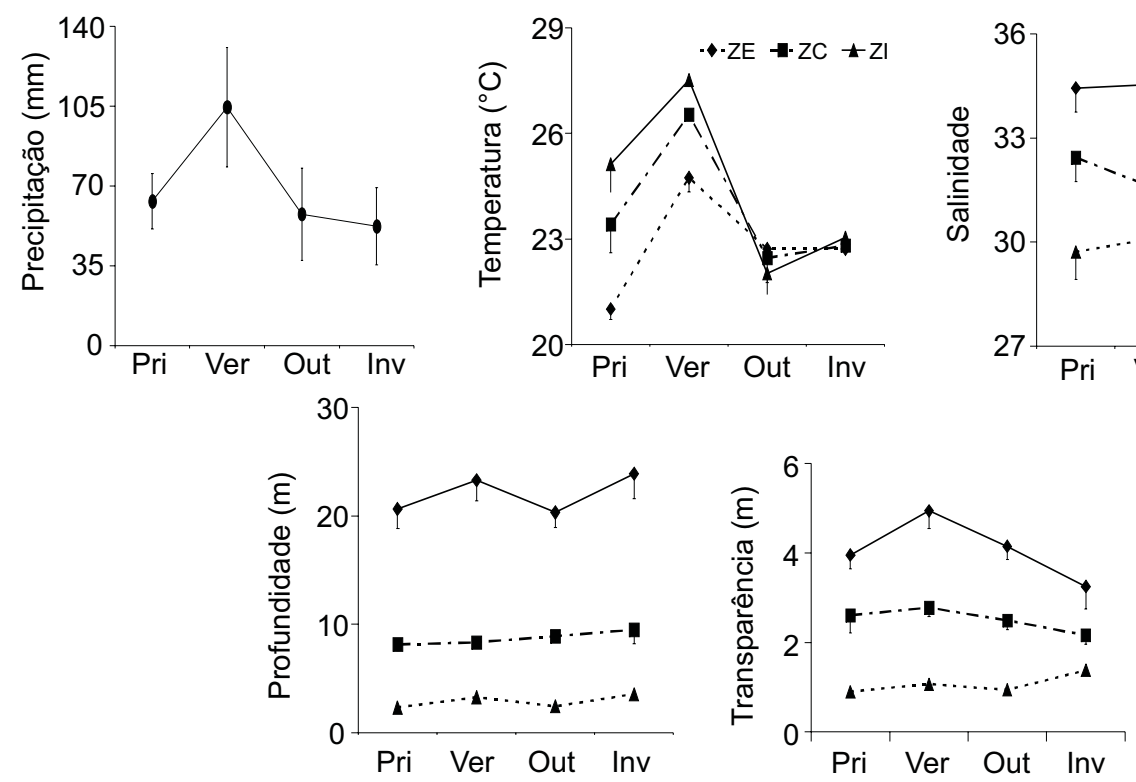

Figura 2. Média e erro padrão (linhas verticais) das variáveis ambientais na Baía de Sepetiba, Rio de Janeiro, 1998/1999. Zonas da Baía: (ZE) zona externa, (ZC) zona central, (ZI) zona interna. Estações do ano: (PRI) primavera, (VER) verão, (OUT) outono, (INV) inverno.

A profundidade apresentou maiores valores médios na zona externa (ZE) em relação à zona interna (ZI) (Fig. 2, Tab. I), sendo essas diferenças detectadas pelo teste de ANOVA (Tab. I).

A transparência apresentou valores médios entre 0,9 e 4,9 metros. Sazonalmente uma tendência de maiores valores foi apresentada somente na zona externa durante o verão, embora o teste ANOVA não tenha detectado diferenças significativas entre as estações do ano, nem interação estação versus zona (Fig. 2). Espacialmente os maiores valores ocorreram na zona externa (ZE) e os menores valores na zona interna (ZI) (Fig. 2, Tab. I).

\section{Distribuição espacial e temporal}

Foram capturados 866 indivíduos, compreendendo um peso total de $118 \mathrm{~kg}$ e, estando freqüentes em 47,2\% das 101 amostragens de arrastos de fundo. Os peixes mediram entre 31 e $293 \mathrm{~mm} \mathrm{C}_{\mathrm{T}}$, sendo representados por jovens e adultos.

Um maior número de indivíduos foi registrado na zona externa em relação às zonas central e interna (Fig. 3, Tab. II). Em relação ao peso também foi registrado este mesmo padrão de maiores abundancias na zona externa. (Fig. 4, Tab. II).
Diferenças nos CPUEs entre as estações do ano não foram significativas para o número de indivíduos. Por outro lado, as CPUEs em peso foram significativamente maiores na primavera quando comparadas com o inverno; o verão e outono apresentaram valores intermediários (Figs 3 e 4, Tab. II).

Relação entre os fatores ambientais e abundância dos peixes

Correlação significativa foi detectada entre a abundância dos peixes e os fatores abióticos de acordo com o coeficiente de correlação de Spearman. Profundidade, salinidade e transparência apresentaram significativas correlações positivas com as CPUEs (indivíduos/arrasto) e (peso/arrasto), enquanto que o peso apresentou correlação negativa com a temperatura (Tab. III).

\section{Período reprodutivo}

Foram examinadas gônadas de 159 indivíduos, sendo 89 machos $(55,98 \%)$ e 70 fêmeas, $(44,02 \%)$. Comparações na proporção sexual por grupo de tamanho, de acordo com o teste do Qui-quadrado $\left(\chi^{2}=12,98\right)$ foram apresentados (Tab. IV). A estrutura de tamanho apresentou diferença significativa na proporção sexual em apenas um único grupo de tamanho $\left(C_{\mathrm{T}}=280 \mathrm{~mm}\right)\left(\chi^{2}=6,0 ; \mathrm{p}<0,01\right)$, com a maior proporção de

Tabela II. Valores e significância de F da Análise de Variância para as comparações de CPUEs para O. ruber na Baía de Sepetiba, Rio de Janeiro, 1998/1999. Zonas da Baía: (ZI) zona interna, (ZC) zona central, (ZE) zona externa.

\begin{tabular}{|c|c|c|c|c|}
\hline Parâmetros & Zona & Estação do Ano & $1 \times 2$ - Interação & Teste de Tukey \\
\hline CPUE indivíduos /arrastos & $45,84^{* *}$ & ns & ns & ZE $>$ ZC, ZI \\
\hline CPUE peso /arrastos & $18,28^{* *}$ & $3,48^{*}$ & ns & $\begin{array}{l}\mathrm{ZE}>\mathrm{ZC}, \mathrm{ZI} \\
\text { primavera }>\text { inverno }\end{array}$ \\
\hline
\end{tabular}

* Significativo ao nível de $95 \%$ de probabilidade $(p<0,05)$; ${ }^{* *}$ significativo ao nível de $99 \%$ de probabilidade $(p<0,01)$. 

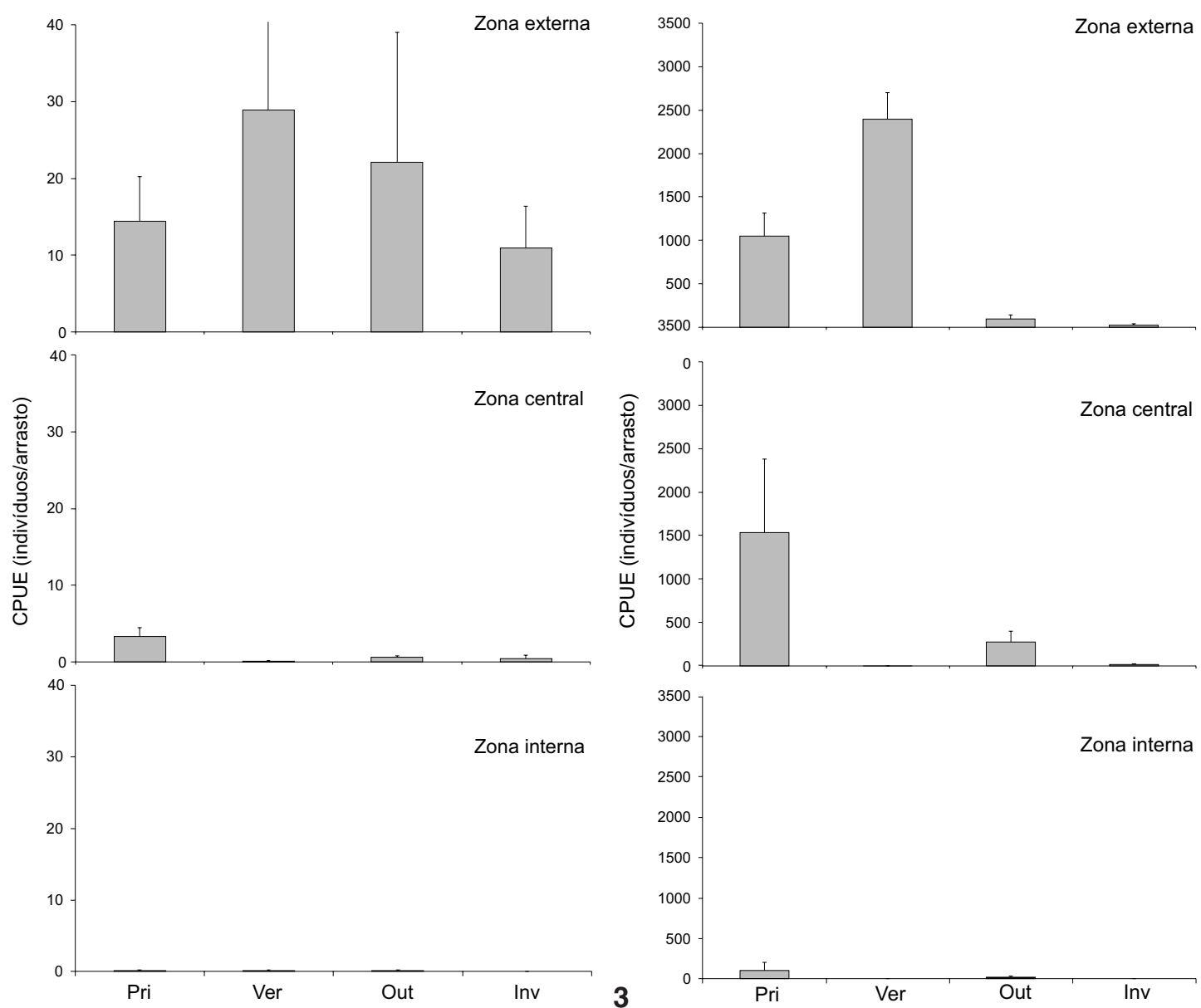

Figuras 3-4. Média e erro padrão (linhas verticais) das Capturas por Unidade de Esforço em número (3) e peso (4) (CPUE - indivíduos/ arrasto) de Orthopristis ruber na Baía de Sepetiba, Rio de Janeiro, 1998/1999. Estações do ano: (PRI) primavera, (VER) verão, (OUT) outono, (INV) inverno.

Tabela III. Coeficiente de correlação não paramétrica (r-Spearman) entre as variáveis ambientais e as CPUEs de O. ruber na Baía de Sepetiba, 1998/1999.

\begin{tabular}{lcc}
\hline Variáveis & CPUE indivíduos/arrasto & CPUE peso/arrasto \\
\hline Temperatura & $\mathrm{ns}$ & $-0,22^{*}$ \\
Salinidade & $0,39^{* *}$ & $0,60^{* *}$ \\
Profundidade & $0,22^{*}$ & $0,24^{*}$ \\
Transparência & $0,21^{*}$ & $0,79^{* *}$ \\
\hline
\end{tabular}

* Significativo ao nível de $95 \%$ de probabilidade $(p<0,05)$;

** significativo ao nível de $99 \%$ de probabilidade $(p<0,01)$.

fêmeas em relação a machos. A população como um todo também não apresentou diferença significativa na proporção sexual. A estrutura de tamanho das fêmeas examinadas variou de 60 a $276 \mathrm{~mm} \mathrm{C}_{\mathrm{T}}$, enquanto os machos variaram de 60 a 260 mm de $C_{\mathrm{T}}$ (Tab. IV).
Não foram detectadas diferenças significativas no fator de condição (K) entre os sexos de acordo com ANOVA ( $\mathrm{p}<$ $0,05)$ (Fig. 5). Apenas as fêmeas apresentaram variações no fator de condição entre os meses amostrados $(\mathrm{F}=2,10, \mathrm{p}<0.05)$ como diferenças significativas entre os maiores valores de junho e agosto e os menores valores de maio (Fig. 5).

Diferenças significativas no índice gonadosomático (IGS) foram encontradas entre os sexos $(F=15,83$. $p<0,001)$ com as fêmeas apresentando maiores IGS. A exemplo do que foi encontrado para $\mathrm{K}$, apenas as fêmeas apresentaram variações significativas no IGS entre os meses ( $F=7,60, p<0,001)$ entre os meses de coleta, com maiores valores entre junho e agosto e os menores de janeiro a maio (Fig. 6).

\section{DISCUSSÃO}

Foi constatado que O. ruber é uma das espécies marinhas que utiliza a riqueza de áreas costeiras semi-fechadas, como baias, durante parte de seu ciclo de vida. Na Baía de Sepetiba, 


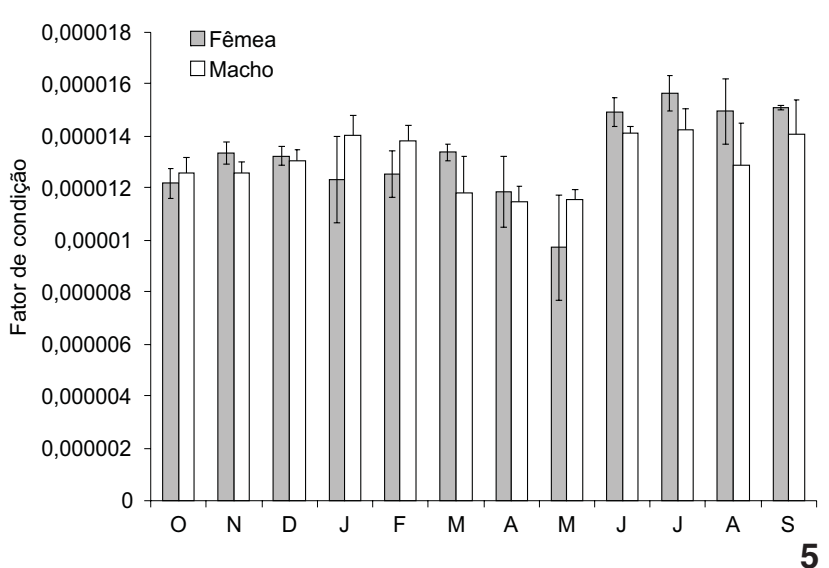

Figuras 5-6. Média e erro padrão (linhas verticais) do fator condição (5) e do índice gonassomatico (6) de Orthopristis ruber na Baía de Sepetiba, Rio de Janeiro, 1998/1999.

Tabela IV. Comparação por classe de tamanho da proporção sexual de O. ruber na Baia de Sepetiba, Rio de Janeiro, 1998/1999. (Fe) Freqüência esperada; $\left(\chi^{2}\right)$ Qui-quadrado.

\begin{tabular}{rccrccc}
\hline Classe & Fêmeas & Machos & $\begin{array}{c}\text { Fêmeas } \\
(\%)\end{array}$ & $\begin{array}{c}\text { Machos } \\
(\%)\end{array}$ & Fe & $\chi^{2}$ \\
\hline 60 & 1 & 1 & 50,00 & 50,00 & 1,0 & 0,00 \\
80 & 2 & 1 & 66,60 & 33,30 & 1,5 & 0,33 \\
100 & 1 & 1 & 50,00 & 50,00 & 1,0 & 0,00 \\
120 & 6 & 5 & 54,50 & 45,40 & 5,5 & 0,09 \\
140 & 4 & 5 & 44,40 & 55,50 & 4,5 & 0,11 \\
160 & 3 & 3 & 50,00 & 50,00 & 3,0 & 0,00 \\
180 & 3 & 6 & 33,30 & 66,60 & 4,5 & 1,00 \\
200 & 6 & 11 & 35,20 & 64,70 & 8,5 & 1,47 \\
220 & 15 & 18 & 45,40 & 54,50 & 16,5 & 0,27 \\
240 & 13 & 22 & 37,10 & 62,80 & 17,5 & 2,31 \\
260 & 10 & 16 & 38,40 & 61,50 & 13,0 & 1,38 \\
280 & 6 & 0 & 100,00 & 0,00 & 3,0 & $6,00 *$ \\
\hline Total & 70 & 89 & 44,02 & 55,98 & 79,5 & 2,27 \\
\hline
\end{tabular}

* Significativo ao nível de $99 \%$ de probabilidade $(p<0,01)$.

suas maiores concentrações ocorreram junto à zona mais próxima do mar, uma sugestão de que condicionantes ambientais (bióticas ou abióticas) no interior da baia podem estar impedindo a penetração destes indivíduos para zonas mais internas. Indivíduos medindo entre 31 a $293 \mathrm{~mm} \mathrm{C}$ formaram a parte da população que usa a Baía de Sepetiba, uma indicação que durante esta parte do ciclo de vida, jovens e adultos desta espécie encontram nesta área condições ambientais favoráveis para seu desenvolvimento. A ausência de indivíduos menores que $30 \mathrm{~mm} \mathrm{C}_{\mathrm{T}}$ pode estar ligada ao fato desta espécie estar utilizando outras áreas como locais de recrutamento, porém não

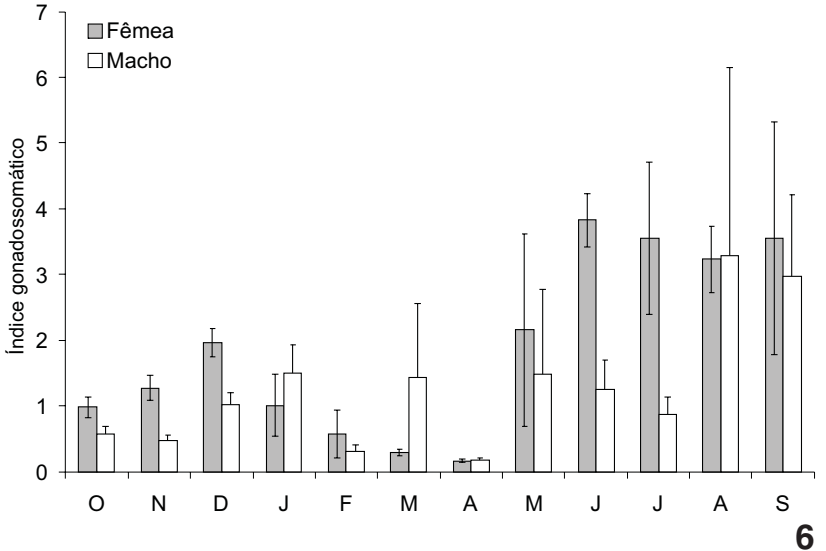
.

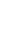

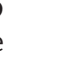


netra na plataforma continental, comprimindo a massa de Águas Costeiras (AC) para o interior dos ambientes semi-fechados da costa sudeste do Brasil. As maiores abundâncias registradas no verão parecem indicar deslocamento destas massas de água sobre a plataforma continental no sentido da zona costeira adjacente, aumentando assim as abundancias destes peixes na zona externa da Baía de Sepetiba. Este padrão sazonal foi mais evidente para os peixes de maior tamanho, indicados pelos picos de biomassa bem definidos, enquanto os indivíduos menores são freqüentes todo o ano.

Um outro fato para a ocorrência de menores abundancia de O. ruber nas zonas mais interiores da Baía de Sepetiba poderia estar ligado à competição com espécies demersais adaptadas ao fundo de baías, como corvinas e bagres. Azevedo et al. (1999) detectaram elevadas abundâncias de bagres marinhos da família Ariidae nas zonas mais internas da Baía, com este mesmo padrão tendo sido detectado por CosTa \& ARAújo (2002) para corvina Micropogonias furnieri. Tais espécies apresentam elevadas densidades na zona interna da Baía de Sepetiba, que similarmente a $O$. ruber, também utilizam áreas próximas ao substrato, se alimentado principalmente de organismos bentônicos da macrofauna (Aguiar \& Filomeno 1995). Portanto, as corvinas e os bagres marinhos poderiam ser competidores potenciais de $O$. ruber, evitando, assim que esta última espécie amplie sua distribuição nas baías. Milagre et al. (2002) observaram que a cabrinha (Prionotus punctatus) também apresenta maiores abundâncias na zona externa da Baía de Sepetiba, e associa este padrão com possível mecanismo para evitar a competição com as espécies abundantes da zona interna. ARAújo et al. (1998) concluíram que a zona externa da Baía apresenta melhores condições ambientais, especialmente menores variações e menores influências de impactos antropogênicos, além de um menor número de competidores quando comparados com a zona interna.

O fator condição $(\mathrm{K})$ permite avaliar as variações das condições gerais do bem estar dos peixes em relação à disponibilidade de alimento (VAzzoler \& VAzzoler 1965). Os investimentos corporais, indicados pelo fator condição, apresentaram variação sazonal pouco marcada entre os sexos. SANTos et al. (2004) determinaram alometria positiva para ambos os sexos de $O$. ruber na Baía de Sepetiba e sugeriram um elevado investimento no crescimento provavelmente um mecanismo para suportar as condições de estresse nesta área. No período de março a maio, nota-se uma diminuição da condição de ambos os sexos, em relação aos outros meses do ano, embora diferenças significativas tenham sido observadas apenas para fêmeas (maiores de junho a setembro). Esta variação parece ser mais associada a variações nos recursos alimentares do que associada ao comportamento reprodutivo, uma vez que os maiores valores do índice gonadosomático para fêmeas ocorreram de junho a agosto. Vianna \& Verani (2002), estudando populações de O. ruber na costa norte do estado de São Paulo, encontraram maiores índice-gonadossomático entre setembro e dezembro, portanto um pouco diferenciado do padrão encontrado na Baía de Sepetiba, onde a reprodução parece ocorrer no inverno. De acordo com BAGENAL (1957) ocorre uma diminuição na condição dos peixes à medida que os indivíduos vão desovando, porém isto não foi verificado na Baía de Sepetiba, quando as menores condições antecederam o período reprodutivo. Os elevados valores de $\mathrm{K}$ registrados para fêmeas de $O$. ruber na Baia de Sepetiba entre junho e setembro, poderiam estar indicando melhores condições alimentares e fisiológicas, e menores estresse na época do inverno e que estes peixes não teriam esta condição influenciada pela reprodução.

\section{AGRADECIMENTOS}

À Universidade Federal Rural do Rio de Janeiro (UFRRJ) pelo apoio de infra-estruturas. Aos meus amigos do Laboratório de Ecologia de Peixes: André (Niterói), Hamilton, Leonardo, Patrícia, Tatiana, Albieri, Tatiana de Mello Lopes e Paulo pela ajuda nas coletas de campo.

\section{REFERÊNCIAS BIBLIOGRÁFICAS}

Aguiar, J.B.S. \& M.J.B. Filomeno. 1995. Hábitos alimentares de Orthopristis ruber (Cuvier, 1830), (Osteichthyes - Haemulidae) na Lagoa da Conceição, SC, Brasil. Biotemas 8 (2): 41-49.

Albaret J.J. 1999. Les peuplements des estuaries et des lagunes, p. 325-349. In: C. LévêQue \& D. Paugy (Eds). Les poissons des eaux continetales africaines: diversité, biologie, écologie et utilization par I'homme. Paris, Éditions de I'IRD, 549p.

Araújo, F.G.; A.G. Cruz-Filho; M.C.C. Azevedo \& A.C.A. Santos. 1998. Estrutura da comunidade de peixes demersais da Baía de Sepetiba, RJ. Brazilian Journal of Biology 58: 417-430.

Araújo, F.G.; M.C.C. Azevedo; M.A. Silva; A.L.M. Pessanha; I.D. Gomes \& A.G. Cruz-Filho. 2002. Environmental influences on the demersal fish assemblage in the Sepetiba Bay, Brazil. Estuaries 25: 441-450.

Azevedo, M.C.C.; F.G. Araújo; A.G. Cruz-Filho; I.D. Gomes \& A.L.M. Pessanha. 1999. Variação espacial e temporal de bagres marinhos (Siluriformes, Ariidae) na Baía de Sepetiba, Rio de Janeiro. Brazilian Journal Biology 59: 443-454.

Bagenal, T.B. 1957. Annual variations in fish fecundity. Journal of the Marine Biological Association of the United Kingdom 36: 377-382

Blaber, S.J.M. 2000. Tropical estuarine fishes - ecology, exploitation and conservation. Cleveland, Blackwell Science, Fish and Aquatic Resources Series 7, 372p.

Costa, M.R. \& F.G. Araújo. 2002. Distribution of Micropogonias furnieri (Pisces, Sciaenidae) in the Sepetiba Bay, Rio de Janeiro, Brazil. Revista de Biologia Tropical 50: 217-225.

Helfman, G.S.; B.B. Collette \& D.E. Facey. 2000. The Diversity of Fishes. Massachusetts, Blackwell Science, 528p.

IsAAC-NAHUM, V.J. \& A.E.A.M. VAZZOLER. 1987. Biologia reprodutiva de Micropogonias furnieri (Desmarest, 1823) (Teleostei, Sciae- 
nidae). 2. Relação gonadossomática, comprimento e peso dos ovários como indicadores do período de desova. Boletim Instituto Oceanográfico 32 (1): 1-16.

LE CREN, E.D. 1951. The lenght-weight relationship and seasonal cycle in gonad and conditions in the perch Perca fluviatilis. Journal of Animal Ecology 20 (2): 201.

Menezes, N.A. \& J.L. Figueiredo 1980. Manual de peixes marinhos do sudeste do Brasil. Teleostei (3). São Paulo, Museu de Zoologia, 96p.

Milagre, R.R.; M.C.C. Azevedo. \& F.G. Araújo. 2002. Distribuição e abundância de Prionotus punctatus (Scorpaeniformes, Triglidae) na Baia de Sepetiba, RJ. Acta Biologica Leopoldensia 24: 173-184.

Moyle, P.B \& J.J. Cесн JR. 1988. Fishes: an introduction to ichthyology. New Jersey, Prentice Hall, $2^{\text {nd }}$ ed., 559p.

Pessanha, A.L.M.; F.G. Araújo; M.C.C. Azevedo \& I.D. Gomes. 2000. Variações temporais e espaciais da comunidade de peixes jovens da Baía de Sepetiba, Rio de Janeiro. Revista Brasileira de Zoologia 17: 251-261.

Rocha, G.R.A. \& C.L.D.B. Rossi-Wongtschowski. 1998. Demersal fish community on the inner shelf of Ubatuba, southeastern Brazil. Revista Brasileira de Oceanografia 46 (2): 93-109.

Rossi-Wongstschowski, C.L.D.B \& E.T. Paes. 1993. Padrões espaciais e temporais da comunidade de peixes demersais do litoral norte do estado de São Paulo, Ubatuba, Brasil. Publicação Especial do Instituto de Oceanografico (10): 169-188.

Santos, A.L.B; A.L.M. Pessanha; M.M. Costa \& F.G. AraúJo. 2004. Relação peso-comprimento de Orthopristis ruber (Cuvier) (Teleostei, Haemulidae) na Baia de Sepetiba, Rio de Janeiro, Brazil. Revista Brasileira de Zoologia 21 (2): 185-187.

VAzzoler, A.E.A.M. 1996. Biologia da reprodução de peixes teleósteos: teoria e prática. Maringá, EDUEM, 169p

Vazzoler, A.E.A.M. \& G. Vazzoler. 1965. Relation between condition factor and sexual development in Sardinella aurita (Cuv. \& Val. 1847). Anais da Academia Brasileira de Ciências 37: 353-359.

ViannA, M. \& J.R. VERANI 2002. Biologia reprodutiva de orthopristis ruber (Teleostei, Haemulidae) espécie acompanhante da pesca de arrasto do camarão-rosa, no sudeste Brasileiro. Atlântica 23 (1): 27-36.

Weinstein, M.P. 1982. Commentary: A need for more experimental work in estuarine fisheries ecology. Journal of Northeast Gulf Science 5: (2): 59-63.

Wootton, R.J. 1989. Introduction: strategies and tactics in fish reproduction, p. 1-12. In: G.W PотTs \& M.N. Wоoтоn (Eds). Fish reproduction: strategies and tatics. London, Academic Press, 410p.

Wootton, R.J. 1992. Fish Ecology. London, Blackie, 212p.

Recebido em 22.XII.2006; aceito em 20.XI.2007. 